\title{
Navigation of a Virtual Exercise Environment with Microsoft Kinect by People Post-Stroke or With Cerebral Palsy
}

\author{
John Hoyle ${ }^{1}$, Justin McCroskey ${ }^{2}$, Lloyd Cooper ${ }^{3}$ and Alan W Eberhardt*1 \\ ${ }^{1}$ Department of Biomedical Engineering, University of Alabama, Birmingham, AL, USA \\ ${ }^{2}$ Lakeshore Foundation, Birmingham, AL, USA
}

${ }^{3}$ PUSH Product Design, Birmingham, AL, USA

Received: 阱 March 27, 2018; Published: 盋April 05, 2018

*Corresponding author: Alan W Eberhardt, Professor and Associate Chair of Education, Department of Biomedical Engineering, Director, Master of Engineering in Design \& Commercialization, University of Alabama, Birmingham, Hoehn 361,1075 13th St. S, Birmingham, AL 35294-4440, USA, Tel: (205) 934-8464; Email: aeberhar@uab.edu

\begin{abstract}
People with physical disabilities have less access to exercise opportunities than their able bodied peers, which contributes to the overall lack of physical activity noted today. In order to help provide engaging active options for this diverse group of people, an Advanced Virtual Exercise Environment Device was designed with adaptable components and an integrated virtual display. Voice commands were used and a custom balance sensing system was created using Velostat to allow users to make choices within a virtual environment. The voice recognition system was tested at various background noise levels and found to be highly accurate within standard conversational ranges for a non-impaired speaker. The balance system was verified for an unimpaired user both while standing and while seated in a wheelchair, though the system was much more responsive for the standing operation mode. A user study with 19 individuals with diverse physical disabilities was performed to obtain feedback on how the various parts of the device functioned for a wide cross section of disabilities. The results showed that the device was functional in all of its components. Initial indications suggested that normal use of the device could provide enough challenge for cardiovascular health maintenance. The device without the accompanying virtual interface was perceived to be generally enjoyable, not boring. Users did not dislike the experience and found it to be more engaging with the virtual interface.
\end{abstract}

Abbreviations: $\mathrm{AVE}^{2} \mathrm{D}$ : Advance Virtual Exercise Environment Device

\section{Introduction}

Physical activity has become dangerously low among all segments of society. In fact, only $21 \%$ of adults have been reported to meet recommended levels of physical activity [1]. As such, beneficial exercise activities should be promoted among all demographics. According to one study, people with physical disabilities made up less than $30 \%$ of the total population, but $40 \%$ of the obese population [2]. Additionally, those with physical disabilities suffered more than their able-bodied peers from a wide array of secondary disabilities including pain, fatigue, depression, and pressure sores [3]. Although exercise could help to improve quality of life and alleviate the presence of many of these secondary disabilities [4-7], those with physical disabilities consistently have not achieved the recommended levels of exercise [8]. A number of factors have influenced these low exercise participation rates, with some of the most critical being cost, lack of motivation, and lack of usable equipment [8-12]. Thus, an effective solution would address the issues of motivation and accessibility.

One common strategy to increase motivation for exercise has been to use active video games or other virtual strategies. There have been a myriad of studies using commercial devices to investigate energy expenditure. Dance Dance Revolution and the Nintendo Wii (Kyoto, Japan) were both popular platforms for study [12-16] and some studies have created custom interfaces for the Microsoft Kinect (Redmond, WA) using specialized code $[17,18]$ A 
limitation of these studies has been that commercial devices are not inclusive technologies, which has necessitated the creation of customized test platforms $[19,20]$. Even in these custom devices, accessibility was normally limited to a single disability group. A more universally applicable device would hold a much greater potential for wide impact.
The current project designed an Advance Virtual Exercise Environment Device (AVE ${ }^{2} \mathrm{D}$, pronounced "av-id"), which combined a custom physical device with a virtual environment to enable and encourage the largest possible number of people to engage in beneficial physical activity, and features high adaptability and a custom interface for an AVG-style experience to increase physical activity among those with physical disabilities.

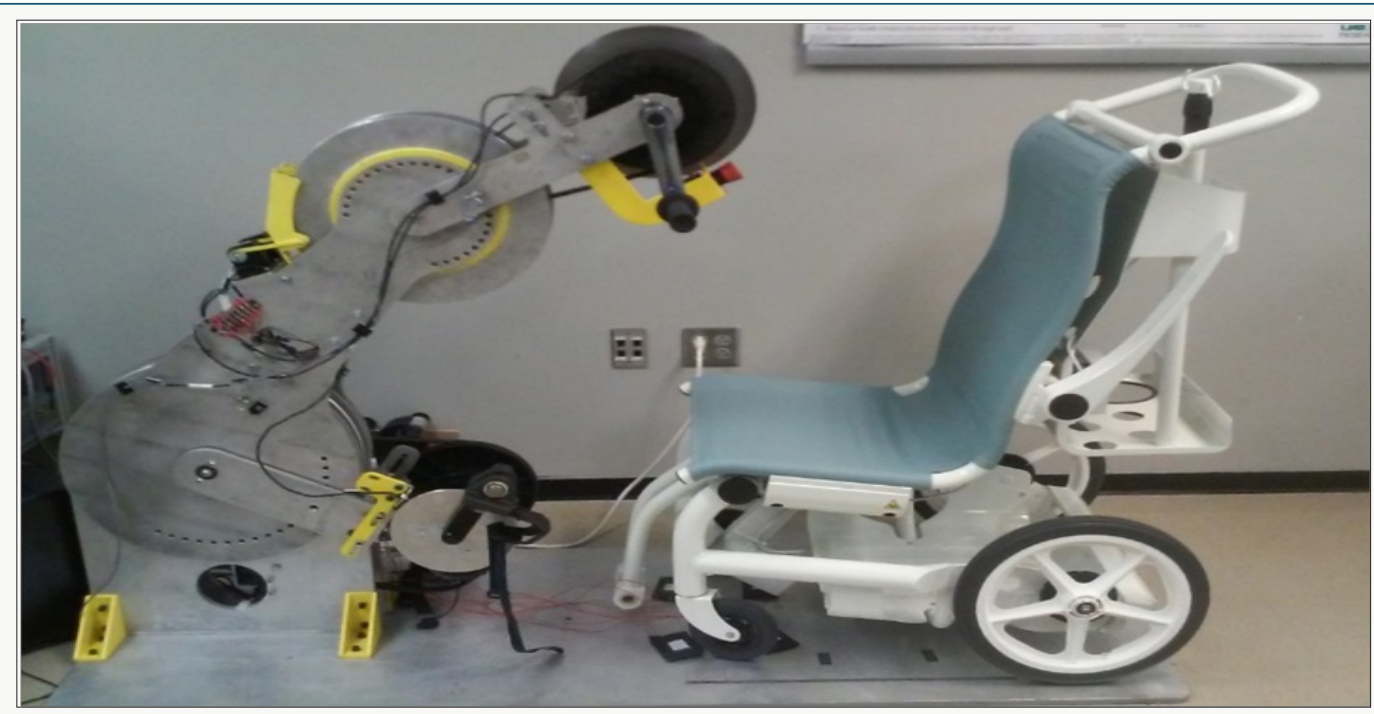

Figure 1: Physical device with upper and lower crank limbs, magnetic flywheel resistance mechanisms. The groove labeled "A" is one of the channels that caused the difficulty in balance sensing. Movi is also displayed on the right side of the figure.

\section{Instrumentation}

The fabricated device (Figure 1) was designed and developed by PUSH Product Design, Birmingham, AL. Exercise is derived through applying energy into crank inputs on both the arm and the leg axes. Resistance is generated through conductive flywheels rotating in a magnetic field. The arms and legs can be operated individually, and the device was created to be used either while standing or seated in a wheelchair. For the user study, users who did not have their own wheelchair completed the seated portion of the protocol in a custom mobility device called Movi, which was also designed by PUSH Product Design. Movi is an electrically powered transportation device for easier transitions between sitting and standing, and was chosen for the study to investigate the utility of the $\mathrm{AVE}^{2} \mathrm{D}$ and Movi as a complete package. Movi weighs more than a normal wheelchair, but was selected to study whether it may make the exercise experience more convenient for people with physical disabilities who do not use a wheelchair.

\section{Resistance Modulation}

Resistance to rotation of the arm and leg axes is generated using eddy currents based on Lenz's Law, which is shown in Equation 1.

$$
\varepsilon=\frac{d \Phi_{B}}{d t}
$$

In this equation, $\varepsilon$ is the electromotive force, $\Phi_{B}$ is the magnetic flux, and $t$ is time. For the present purpose, this equation means that an electromotive force was generated to oppose a changing magnetic flux. The resistance mechanism in the $\operatorname{AVE}^{2} \mathrm{D}$ used a conductive flywheel rotating in a magnetic field to induce the flux, which generated a resistive torque that opposed the direction of motion. Analysis of these types of brakes has shown that the resistance is dependent on several factors, most importantly angular velocity, magnetic field strength, and area that the magnet covered on the disc [21]. Only two of these factors change during use of $\mathrm{AVE}^{2} \mathrm{D}$ : disc angular velocity and magnet overlap. Since the angular velocity is user controlled, the resistance level is adjusted through magnet position modulation.

The magnet position control is controlled using an Arduino Uno microcontroller and a potentiometer combined with a servomotor as shown in (Figure 2A). The magnet is connected to the motor, which enables reading the potentiometer data as a measurement of the magnet position. The Arduino reads the current position, compares it to the desired position, and drives the servomotor to the desired position using a PID-style algorithm. The device uses three of these resistance systems: one for each individual arm and one for the legs together.

\section{RPM Sensing}

RPM is measured through the use of proximity sensors and 12-tooth gears. The system is shown in (Figure 2B). As each tooth passes by the appropriate sensor, the event is recorded. The number of teeth that pass in a given amount of time allows RPM to be calculated. These values are updated dynamically and used to control the virtual interface. 


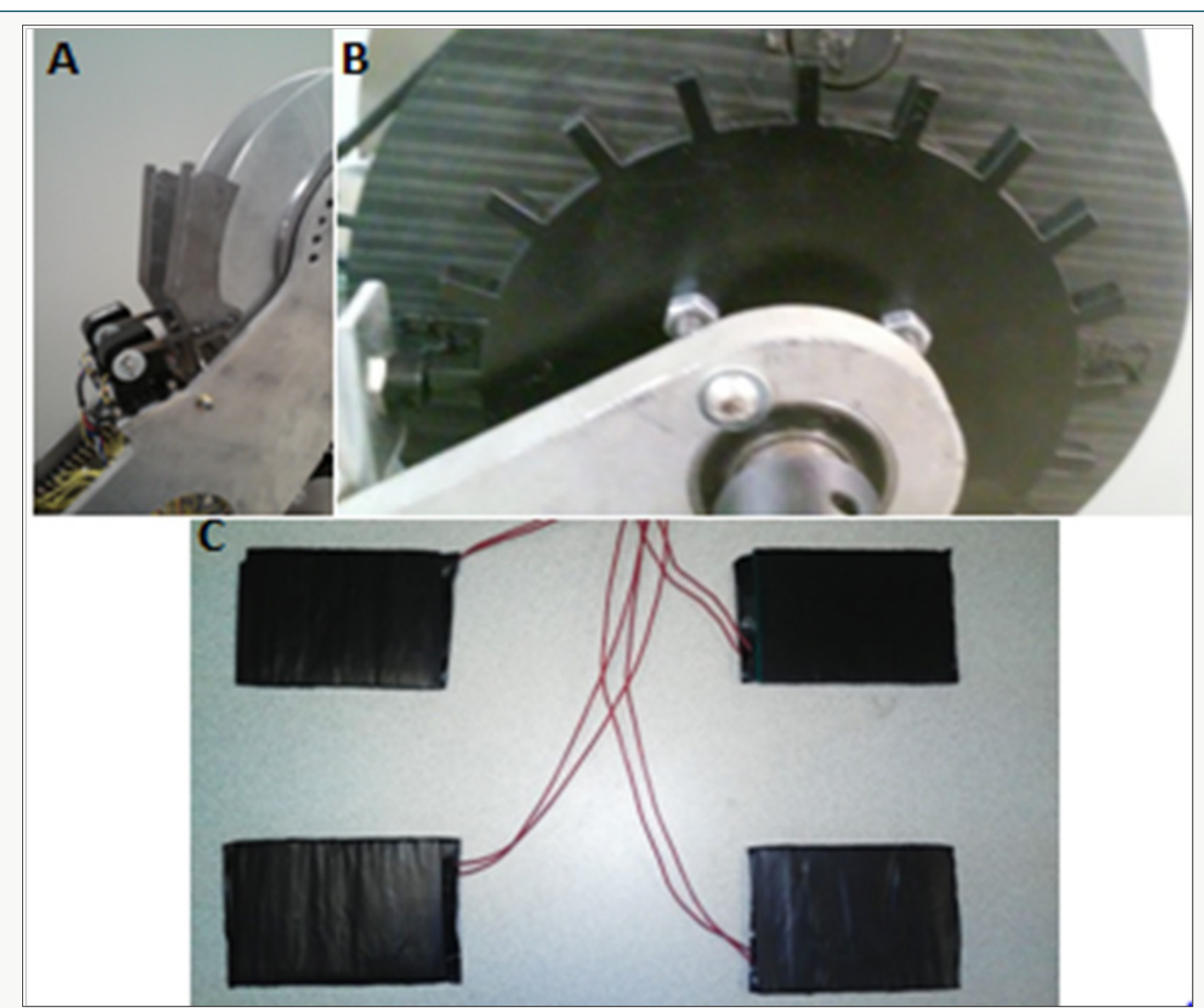

Figure 2: A) Magnetic resistance components where the servomotor/potentiometer combo (black box) moved the magnets (dark gray) closer or farther from the flywheels. B) The gear teeth passed by a proximity sensor to provide RPM readings. C) Velostat pads used for balance sensing. For standing conditions, they would be placed beneath the heel and toe of each foot. For seated conditions, they would rest under the front and back wheels of the wheelchair or mobility device.

Balance Sensing: The balance system uses four pads made of Velostat (3M, Maplewood, MN) wired in series to a resistor to create simple voltage dividers. These pads are shown in (Figure $2 \mathrm{C}$ ). The pads are arranged so that they rest under the heel and toe of each foot for standing users and under the front and back wheels of seated users. The voltage after the Velostat and before the resistor is measured on an Arduino, the differences horizontally and vertically

are calculated, and these values are passed to the main computer. The values from each pad when the user is at a neutral position are stored and used as a calibration factor. Velostat behaves so that an increased voltage reading for each pad correlates to increased pressure on that pad, which provides an indirect measurement of relative pressure when compared to the stored neutral values.

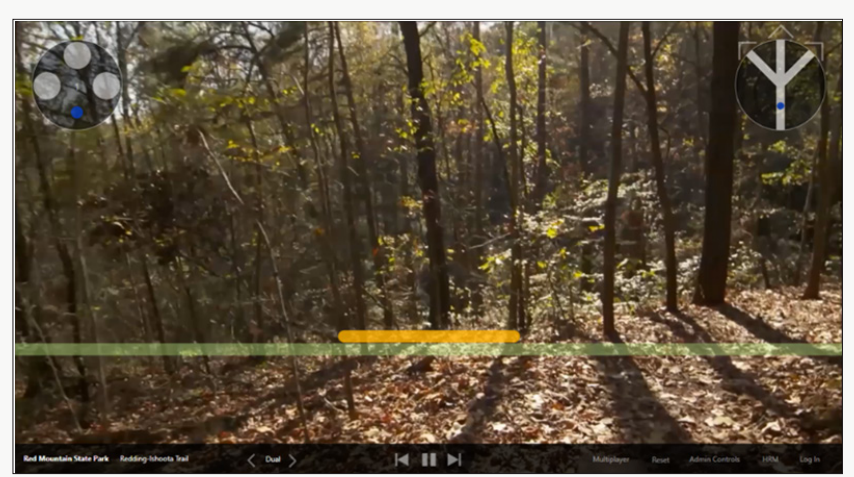

Figure 3: Virtual display with current position, along trail, and continuation choices in the top right corner; balance information in the top left corner; option bar along the bottom; and heart rate information displayed. The green bar represents the set target range and the ellipse represents the current output. The current output ellipse changes colour to represent whether the current effort is low (shades of purple and blue), in the target range (green), or high (shades of orange and red). 
Main Display: The main purpose of the display is to provide a platform for entertainment and encouragement during exercise, which is accomplished using a hiking trail through a wooded park (Figure 3). The entire display was created in Visual Studio 2015 in the C\# language, and is hosted on a computer connected to the physical device via a USB connection. This computer loads a video and displays it as the central part of the screen. The computer receives information on exercise RPM and modulates the play rate of the video to be proportional to the exercise speed. The display reads resistance settings from a text document, compares the current video time with the resistance specifications and sends updated settings when appropriate. These resistance settings are specified so that increasing resistance corresponds to an increase in the grade of the displayed path and decreasing resistance similarly corresponds to a downhill slope. The mean resistance can be adjusted based on user comfort. This means that all resistance levels are shifted higher or lower, but the resistance is still automatically adjusted to reflect the terrain.

Whenever a fork comes up in the path, the user can select which option to take: left, right, or straight. Although clicking with a mouse in designated areas is a supported method of interaction with the display, it is not the preferred mode as it interrupts the exercise experience. Choices can also be made through arrow button presses, voice commands, and balance sensing. The voice recognition uses Microsoft Speech 11.0 to access the default computer device for voice recognition. This project builds on the observations of previous work [22] to make all voice commands multiple syllables to give a wider sample for recognition and to use a desktop microphone rather than the Kinect's onboard microphone.

Heart Rate Monitoring: The interface also has the ability to identify and connect to Bluetooth Low Energy heart rate devices. Once a heart rate monitor is connected, the display automatically updates to show physiologic target intensity ranges. The computer uses the participants' age to calculate percent of maximum heart rate $\left(\% \mathrm{HR}_{\max }\right)$ as a measure of exercise intensity. This value is calculated as shown in Equation 2.

$$
\% H R_{\max }=\frac{H R}{H R_{\text {max }}} * 100 \%
$$

Many studies have estimated HRmax using the equation $\mathrm{HR}_{\text {max }}$ = 220 - age. More recently Tanaka et al analyzed the results from a wide variety of people and revised the equation to $\mathrm{HR}_{\max }=208$ - .7*age, which was the equation used in the present study. The computer display shows a target bar at a desired $\% \mathrm{HR}_{\max }$ as well as the current $\% \mathrm{HR}_{\max }$ level. This display is shown as the bar across the screen in Figure 3, and provides the user extra motivation as well as confidence that their efforts will contribute to long term cardiovascular health. The device, its features, and the code behind it all were described in more detail by Hoyle in 2017.

\section{Methods}

Prior to execution of the study, baseline recognition rates for the voice recognition were determined in a test performed by the lead author at different background noise intensities. The lead author spoke a phrase randomly selected by the computer from the dictionary of the device ( $\sim 50$ phrases) and the number of attempts required before recognition was recorded. A sound system playing crowd noise was used to vary the background noise levels from 43 to $70 \mathrm{dBs}$. One hundred phrases were completed at each decibel level, because this number of phrases provided a strong basis for comparison without requiring excessive repetitions.

The balance system was also tested while standing and while seated in a wheelchair to investigate the ability of the system to operate effectively for the lead author-an able-bodied user. This test used a custom interface that generates a target at a random position and the user attempts to shift his weight to get the circle representing his center of weight into the target area and hold it for a full second (Figure 4A). One hundred iterations of this test were performed while standing, and another hundred while seated. A MATLAB script was used to calculate the time and hand path ratio (HPR). HPR is simply the ratio of distance traveled to straight line distance, and has previously been taken as a measure of movement efficiency [23]. The display and the MATLAB output can be seen in (Figure 4B).

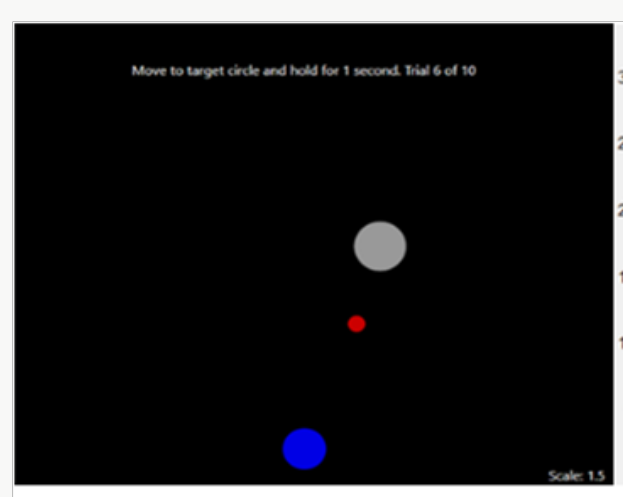

(a)

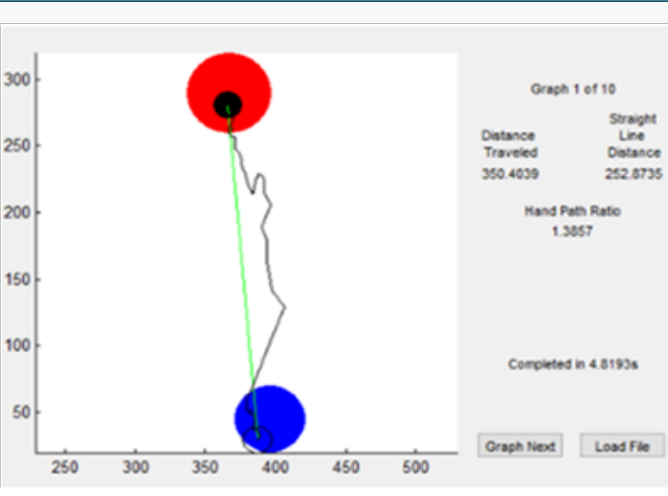

(b)

Figure 4: A) The user interface during a test with target (gray), start (blue), and current position (red). B) MATLAB breakdown of a trial with the path information graphed and the calculated values on the side. These number were automatically exported to Excel for reference later. 
With these basic diagnostics established, 19 users with physical disabilities were recruited to test the device in a protocol that was approved by the University Institutional Review Board (protocol number X161107003). The demographics of the cohort can be found in (Table 1). This diverse group was recruited to test one of the main objectives of this device: to serve as wide a range of people as possible regardless of disability. After an introduction to the device and a brief calibration to each individual's strength, users were asked to use the device in four three minute sessions. Table 1: Demographic information of the participants.
The first two were without the interface-once with arms and legs simultaneously and once with only arms. The second two sessions were the same except with the addition of the virtual interface. The users were able to choose whether they wanted to stand or sit during the trials with arms only. Wheelchair users completed the study entirely from their own chair, but other participants used Movi. Non-ambulatory participants only completed the conditions without the legs.

\begin{tabular}{|c|c|c|c|}
\hline Participants & Age & Race & Disabilities \\
\hline 19 (9 Female) & $51.6 \pm 16.6$ & $\begin{array}{c}\text { 12 White, } 7 \\
\text { African American }\end{array}$ & $\begin{array}{c}\text { Cerebral Palsy }(\mathrm{n}=4,1 \mathrm{~W}), \text { Stroke }(\mathrm{n}=2,1 \mathrm{~W}), \text { Parkinson's ( } \mathrm{n}=2 \text { ), Neurofibromatosis (W), } \\
\text { Multiple Sclerosis, Spinal Cord Injury, Knee, Transverse Myelitis (W), Amputee (W), Spina } \\
\text { Bifida (W), Polio, Balance Complications, Paraplegia (W) Fibromyalgia }\end{array}$ \\
\hline
\end{tabular}

W indicates a wheelchair user.

Participants were initially asked to navigate the path choices on the display through both voice recognition commands and Velostatmediated leaning. After these initial attempts, the users were free to navigate however they chose. The voice commands used in this test period were only the ones for navigation: "AVE2D Left" for turning left, "AVE2D Right" for turning right, and "AVE2D Straight" or "AVE2D Forward" to continue straight ahead. Participants were given a questionnaire to provide feedback on rate of perceived exertion (RPE) [24] and whether they enjoyed, were bored by, or disliked the activity on a Likert scale (1=strongly disagree, $5=$ =strongly agree) for the device with and without the virtual interface. The questionnaire results presented here were focused on the trials with arms only, since not all participants had adequate leg function to participate in the trials with arms and legs combined. Additionally, four users were outfitted with a heart rate monitor to gather initial data on the possible cardiovascular implications of this device.

\section{Results}

\section{Voice Recognition}

The results for the initial diagnostic tests of the voice recognition are shown in (Figure 5A). During the $55 \mathrm{~dB}$ trial, a computer malfunction caused the microphone to stop responding, which increased the attempts/recognition; therefore, these data were omitted from subsequent analysis. Levene's test showed that the variances were unequal $(\mathrm{p}<0.05)$, but Welch's Test showed no statistical difference in the means. Although the 65 and $70 \mathrm{~dB}$ conditions produced higher attempts/recognition than the others, they did not reach statistical significance. This testing verified that the updated phrases and microphone system could effectively recognize voice commands for the lead author. Thus, the user study could focus on evaluating the utility for people with physical disabilities, unaffected by secondary factors like background noise.

\section{Balance Sensing}

The balance testing results are shown in Figure 5B. In the standing and seated conditions, the lead author was able to consistently complete the task in a relatively quick manner.
However, using the balance system while standing was far more efficient than while seated in the wheel chair. Statistically, t-tests showed that the standing condition was faster and more efficient ( $p<0.005$ for both) than the comparable results while seated. This protocol combined with the voice recognition results sought to establish that the device did function properly for an experienced user. Next, it fell to the user study to investigate the system for usability within the target population.

\section{User Tests}

Two users did not attempt to use the voice recognition system to make path choices due to speech patterns that the study supervisor believed would render the voice recognition software useless. Of the remaining 17 participants more than half (nine) demonstrated an ability to give recognizable commands extremely consistently (no more than one missed recognition in every three attempts). Another three participants struggled with one of the commands, but achieved success with the other two options. The remaining five participants struggled substantially with achieving recognition on at least two of the commands. The overall recognition rates are shown in Figure 5C. During testing only ten of the participants were able to test the balance controls. This lack was caused by a channel cut into the base plate of the AVE2D to allow for an adjustable piece to move freely (Figure 1). Unfortunately this channel often times lined up with the wheel placement for users in the seated trials. Since the Velostat pads needed to be placed under the wheels, the channel caused there to be a stress discontinuity and rendered the balance readings unusable. This was not a problem for users who were standing, but only six participants were able and chose to do so. The results (Figure 5C) while standing were excellent with five of the six navigating with only one missed navigation. Consistent with the validation results, the seated control was less effective. Three of the ten users were able to navigate at $80 \%$ success or higher, four at $50 \%$, and the remaining three struggled to achieve consistent control. There were also four unintentional selections made while in the seated operation mode and none for standing operation. 


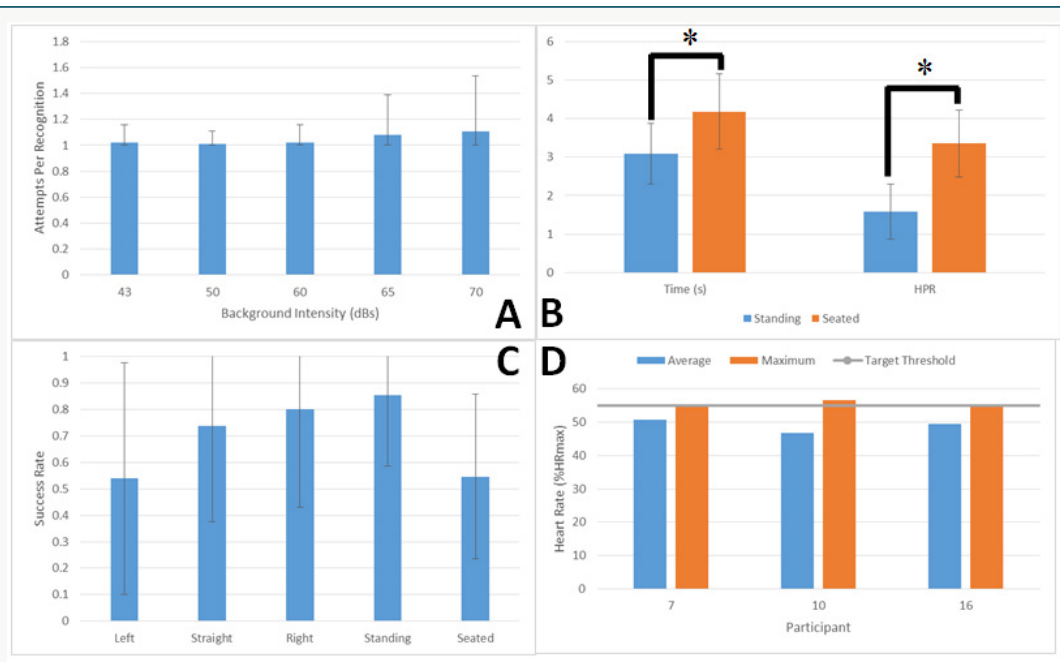

Figure 5: A) Voice recognition results +/- standard deviation for various background interference levels. B) Time and HPR results + /- standard deviation for both standing and seated trials. *Denotes significant differences between the standing and seated operation modes. C) Voice recognition and balance results +/- standard deviation for user study. D) Average and maximum heart rates for the individuals using the device with the virtual interface.

There were four participants who agreed to operate the device while fitted with a heart rate monitor. Other than an indicator of current heart rate relative to $60 \% \mathrm{HRmax}$, there were no changes in the protocol or display. The participants were allowed to operate the device at their own speed and the resistance was changed automatically according to their calibrated strengths and the terrain displayed on the monitor in front of them (Figure 5D) shows the mean and maximum values for each of the participants. All of the users were able to achieve a maximum heart rate above $50 \%$ of their age predicted maximum, and the averages were all near this same benchmark; however, these values were below the recommendation of $55 \% \mathrm{HR}_{\max }$ made by Pollock et al. Four users provided these heart rate measurements, but only three produced valid data sets. The invalid trial resulted in readings that were much

Table 2: RPE and questionnaire results for the user study. too high $\left(>100 \% \mathrm{HR}_{\max }\right)$ for the exercise, the amount of time spent, and the reported RPE, so this trial was not included in the reported heart rate results.

The questionnaire results demonstrated that the participants found the experience generally enjoyable, not boring, and did not dislike the device both with and without the virtual interface. Paired t-tests were used to evaluate whether there was a difference between the user perceptions of the device with and without the virtual interface as expressed on the questionnaire. None of the categories reached significance except for boredom. The participants felt significantly less bored with the interface than without $(\mathrm{p}<0.001)$. The average RPE was higher for users while using the interface, but did not reach significance $(p=0.087)$. The averages and standard deviations can be found in Table 2 .

\begin{tabular}{|c|c|c|c|c|c|c|c|}
\hline \multicolumn{3}{|c|}{ Without Virtual Interface } & \multicolumn{4}{c|}{ With Virtual Interface } \\
\hline RPE & Enjoyed & Bored & Disliked & RPE & Enjoyed & Bored & Disliked \\
\hline $11.31 \pm 2.00$ & $3.95 \pm 1.03$ & $2.37 \pm 0.68$ & $2.21 \pm 0.85$ & $12.42 \pm 1.87$ & $3.89 \pm 1.10$ & $1.63 \pm 0.68$ & $1.89 \pm 0.99$ \\
\hline
\end{tabular}

\section{Discussion}

Compared to previous results [22] the calibration testing indicated that longer phrases and a microphone located closer to the speaker were effective methods of improving recognition. The lack of significant drop off in recognition with increasing background noise was somewhat interesting. In fact, the consistent recognition up to $70 \mathrm{dBs}$ promised success for users in most environments that they are likely to encounter while using the AVE2D, since this was well above the upper limit of sound intensity of normal conversation [25]. While there was a general increase in the recognition rates with increasing background noise, the 70 $\mathrm{dB}$ condition still only required 1.18 attempts per recognition. This average was much better than the actual user tests from the participant study, which was performed at low background noise.

This suggested that background noise should be much less of a factor on recognition effectiveness than factors associated with individual participants.

The initial testing of the balance system was simply a means of verifying the utility of the product under ideal conditions with a trained user. Statistically, the standing mode of operation was better in both time and HPR. This result was expected due to the implementation complications for reading data from a user in a wheelchair. The wheelchair distributed the weight over a wider base and also added enough additional weight on the sensors to noticeably lower the available resolution of the readings. Additionally, the envelope of the chair made it more difficult to lean to the sides, further complicating the process of gathering useful readings. In the test, the lead author navigated to and held their 
position within the target for a full second to achieve a selection. Thus, the time results indicated that while standing, the user took just over two seconds to reach the target on average and more than three while in the wheelchair. The more significant difference in results came from measuring the efficiency of the movement. The user took a path about 50\% longer than the ideal path while standing, but more than three times longer while in the wheelchair. As mentioned previously this was the expected result, and the system could use improvement in the wheelchair mode; however, the presented Velostat-based balance sensing system could have some powerful implications. The ability to accommodate both users who can stand on their own and those who are seated in a wheelchair is a feature that is not shared by other systems.

For example, studies that have recruited people with physical disabilities for testing the Wii balance board have been limited to higher functioning individuals within their classification Thus; it fell to the user study to verify whether this system could provide wider functionality for the actual target population. Out of the 19 participants recruited, 17 attempted using the voice commands to make path continuation choices. The average success rate for voice recognition was far below that of the validation protocol at 1.77 attempts/recognition overall. However, there were several factors that contributed to this difference. First, the participants were not given much time to become acquainted with the voice recognition software, so they were not able to learn the optimal ways of enunciating to enhance success. Second, the recognition percentages disproportionately represent those who were less successful at being recognized, because a missed recognition necessitated another attempt which could compound the number of missed attempts. Third, some users were not impaired in their speech but simply spoke in a way that was not recognizable to the implemented algorithm. Although it was demonstrated several times in the device introduction, some users were not sure how to pronounce the name of the device, which further complicated pronunciation. The most common issue for these users was making the "D" sound at the end of "AVE2D" and the "T" sound in "Left", "Straight", and "Right". This was demonstrated by participant 14 who had two correct recognitions out of six attempts to select the straight continuation. The four missed recognitions all came from trying to say "AVE2D Straight", but the participant was recognized on the first attempt when switching to "AVE2D Forward".

Some of these issues could be reduced by adding a training protocol at the beginning so that the voice recognition software could be calibrated to each individual's speech patterns, or by refining the phrase dictionary to reduce difficult phonetic combinations. Regardless, more than half of the participants still demonstrated a consistent ability to successfully utilize the voice recognition. Among these users, there were no misrecognition errors in the trials where the algorithm selected the incorrect continuation. The majority of failed attempts occurred when the microphone did not report hearing anything at all. Thus, moving the microphone closer to the user or using a more sensitive microphone could also help to increase recognition consistency.

One of the limitations of this study was the lack of data collected for the balance system from the user trials. It was unexpected that the wheels would line up with the channel and preclude the use of the system in so many cases. Although success was the only variable measured for the balance system in the user trials, the standing results were extremely promising. All but one user were highly successful in making path choices under this setup. The participant who did struggle to control the system did not understand that it was more effective to control center of balance by leaning at the hips rather than upper body. It is likely that more experience with the system could have helped to alleviate this confusion. For the seated results, the overall success rate was just below 55\%. Considering that the majority of these tests were run in the Movi, which the balance system was not designed for, the results were fairly promising. Although the Movi provided useful functionality, it was heavier than a normal wheelchair and provided less sensitive readings than expected.

The lack of sensitivity required a scaling factor to be added, which lowered the fine control and contributed to the multiple incorrect selections made across the trials. Additionally, the shifting of the chair as the user pedaled with their feet tended to cause a high amount of jitter in the response of the sensors, which required participants to stop pedaling with their feet before trying to lean into a selection. While compelling as a complete package from a single manufacturer, the Movi combined with the current device construction did not provide ideal functionality for the balance system. It may be advisable to use a normal wheelchair for seated operation in future tests; however, the evidence suggests that this Velostat system could be useful in the future, but it needs to be improved in order to make it more sensitive for users while seated.

The minimum requirement for cardiovascular health has been given to be 55\%HRmax [22]. AVGs and other interactive exercise strategies have consistently struggled to push users into a beneficial cardiovascular. The small amount of time each participant spent actually exercising combined with the relatively few participants who provided heart rate readings made any hard conclusions about the exercise intensity derived from using the device impossible [26]. Although this study was neither exhaustive in data collection nor lengthy enough to constitute a true exercise study, the heart rate data did give an indication that prolonged use could potentially enable users to reach beneficial exercise levels. Of the three trials that produced usable data, all of the users had a maximum heart rate within the target range, and an average heart rate above $45 \%$ HRmax.

Two of the users had an average heart rate near 50\%HRmax. The resistance settings used for this study were calibrated to be on the lower side of what would be suggested for true exercise usage, so increased time and resistance could likely produce high enough 
heart rates for long term health benefits. Rate of perceived exertion (RPE) has been verified to be correlated to heart rate [24], however, the participants who provided the heart rate measurements had CP, polio, and stroke respectively, which could impact their perception of the difficulty. The values for RPE given by these participants tended to be high compared to the heart rate measured, which suggested that those with physical disabilities may feel more strain during exercise than their able bodied peers for comparable levels of exertion.

Taking into account all of the participants, they reported generally higher RPE with the virtual display than without. Since the conditions with the virtual display came second, it is possible that order impacted the responses given by the participants. The difference was not significant in any case, and the trial periods were only three minutes long. According to the questionnaire, the virtual interface did not change the participants' enjoyment of the device. There was no difference between the rating of dislike towards the experience both with and without the virtual display [27]. In fact, both with and without the display the experience was generally enjoyed and participants did not agree with the statement that they disliked the experience. The virtual interface did in fact reduce the perception of boredom. Taken together, these results provided evidence that this device coupled with the virtual feedback elements could encourage consistent physical activity for those with physical disabilities.

The current study demonstrated that a group of people with diverse disabilities were able to use the device and have a positive experience. Future work should improve the balance system to make it more consistent for those in a wheelchair and solve to placement problem [28]. The current physical device is a prototype test platform, so based on the results of the study; the channel cut into the base could be changed to remove the issue experienced in the user tests. Additionally, a study with longer exercise periods should be completed to better analyze the cardiovascular implications of the device and its interface.

\section{Conclusion}

The AVE2D, virtual interface, voice recognition, and balance sensing have demonstrated the desired functionality within the target population. This novel device has a level of adaptability that shows promise for a wide variety of functional needs. The reported perceptions associated with the experience support the assertion that this device coupled with its virtual interface could encourage prolonged use. Initial results suggest that the device could provide an effective exercise opportunity for people with physical disabilities, though more testing is required.

\section{Acknowledgement}

Project supported by RERC RecTech funded by the National Institute on Disability, Independent Living, and Rehabilitation Research (NIDILRR grant number 90RE5009-01-00). NIDILRR is a center within the Administration for Community Living (ACL), Department of Health and Human Services (HHS). The contents of this publication do not necessarily represent the policy of NIDILRR, ACL, HHS, and you should not assume endorsement by the federal government. Thanks to Lloyd Cooper from PUSH Product Design and the entire UAB/Lakeshore Collaborative staff for their continuing support.

\section{References}

1. Centers for Disease Control and Prevention (2014) Facts about Physical Activity.

2. Armour BS, Courtney Long EA, Campbell VA, Wethington HR (2013) Disability Prevalence Among Healthy Weight, Overweight, and Obese Adults. Obesity 21(4): 852-855.

3. Centers for Disease Control and Prevention (2016) Related Conditions.

4. Carlson JE, Ostir GV, Black SA, Markides KS (1999) Disability in Older Adults 2: Physical Activity As Prevention. Behavioral Medicine 24(4): 157.

5. Freiberger E, Sieber C, Pfeifer K (2011) Physical Activity, Exercise, and Sarcopenia-future Challenges. Wmw Wiener Medizinische Wochenschrift 161(17): 416-425.

6. Heath GN, Fentem PH (1997) 8 Physical Activity Among Persons With Disabilities-a Public Health Perspective. Exercise and Sport Sciences Reviews 25(1): 195-234.

7. Rimmer JH, Riley B, Creviston T, Nicola T (2000) Exercise Training in a Predominantly African-american Group of Stroke Survivors. Medicine and Science in Sports and Exercise 32(12): 1990-1996.

8. Boslaugh SE, Andresen EM (2006) Correlates of Physical Activity for Adults with Disability. 30(2): 5-16.

9. Barfield JP, Malone LA (2013) Perceived Exercise Benefits and Barriers Among Power Wheelchair Soccer Players. Journal of Rehabilitation Research and Development 50(2): 231-238.

10. Jaarsma EA, Dijkstra PU, Geertzen JHB, Dekker R (2014) Barriers to and Facilitators of Sports Participation for People With Physical Disabilities: A Systematic Review. Scandinavian Journal of Medicine \& Science in Sports 24(6): 871-881.

11. Rimmer JH (2005) The Conspicuous Absence of People With Disabilities in Public Fitness and Recreation Facilities: Lack of Interest or Lack of Access? American Journal of Health Promotion 19(5): 327-329.

12. Rimmer JH, Wang E, Smith D (2008) Barriers Associated With Exercise and Community Access for Individuals With Stroke. Journal of Rehabilitation Research and Development 45(2): 315-322.

13. Devereaux J (2012) Comparison of Rates of Perceived Exertion Between Active Video Games and Traditional Exercise: Original Research. International Sport med Journal 13(3): 133-140.

14. Graves LE, Stratton G, Ridgers ND, Cable NT (2008) Comparison of Energy Expenditure in Adolescents When Playing New Generation and Sedentary Computer Games: Cross Sectional Study.

15. Tan B, Aziz AR, Chua K, Teh KC (2002) Aerobic Demands of the Dance Simulation Game. International Journal of Sports Medicine 23(2): 125129.

16. Unnithan VB, Houser W, Fernhall B (2006) Evaluation of the Energy Cost of Playing a Dance Simulation Video Game in Overweight and Nonoverweight Children and Adolescents. International Journal of Sports Medicine 27(10): 804-809. 
17. Gerling KM, Kalyn MR, Mandryk RL (2013) Kinect Wheels: Wheelchairaccessible Motion-based Game Interaction. In Chi'13 Extended Abstracts on Human Factors in Computing Systems pp. 3055-3058.

18. Sato K, Kuroki K, Saiki S, Nagatomi R (2015) Improving Walking, Muscle Strength, and Balance in the Elderly With an Exergame Using Kinect: A Randomized Controlled Trial. Games for Health Journal 4(3): 161-167.

19. Hernandez HA, Graham TC, Fehlings D, Switzer L, Ye Z, et al. (2012) Design of an Exergaming Station for Children With Cerebral Palsy. In Proceedings of the Sigchi Conference on Human Factors in Computing Systems pp. 2619-2628.

20. Widman LM, Mcdonald CM, Abresch RT (2006) Effectiveness of an Upper Extremity Exercise Device Integrated With Computer Gaming for Aerobic Training in Adolescents With Spinal Cord Dysfunction. The Journal of Spinal Cord Medicine 29(4): 363-370.

21. Wouterse JH (1991) Critical Torque and Speed of Eddy Current Brake with Widely Separated Soft Iron Poles. In Iee Proceedings B-electric Power Applications 138(4): 153-158.

22. Pool SM, Hoyle JM, Malone LA, Cooper L, Bickel CS, et al. (2016) Navigation of a Virtual Exercise Environment With Microsoft Kinect by People Post-stroke or With Cerebral Palsy. Assistive Technology 28(4): 225-232.
23. Zimmerli L, Krewer C, Gassert R, MüllerF, Riener R, et al. (2012) Validation of a Mechanism to Balance Exercise Difficulty in Robot-assisted Upperextremity Rehabilitation After Stroke. Journal of Neuroengineering and Rehabilitation 9(1): 6

24. Borg GA (1982) Psychophysical Bases of Perceived Exertion. Med Sci Sports Exerc 14(5): 377-381.

25. Daniel E (2007) Noise and Hearing Loss: A Review. Journal of School Health 77(5): 225-231.

26. Hoyle J (2017) Design, Testing, and Implementation of Controls and Interface for an Adaptable Exercise Device for People with Physical Disabilities.

27. Pollock ML, Gaesser GA, Butcher JD, Després JP, Dishman RK, et al. (1998) Acsm Position Stand: The Recommended Quantity and Quality of Exercise for Developing and Maintaining Cardiorespiratory and Muscular Fitness, and Flexibility in Healthy Adults. Med Sci Sports Exerc 30(6): 975-991.

28. Tanaka H, Monahan KD, Seals DR (2001) Age-predicted Maximal Heart Rate Revisited. J Am Coll Cardiol 37(1): 153-156.

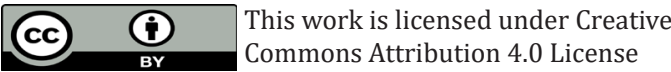

To Submit Your Article Click Here: Submit Article

DOI: $10.32474 /$ OAJBEB.2018.02.000130

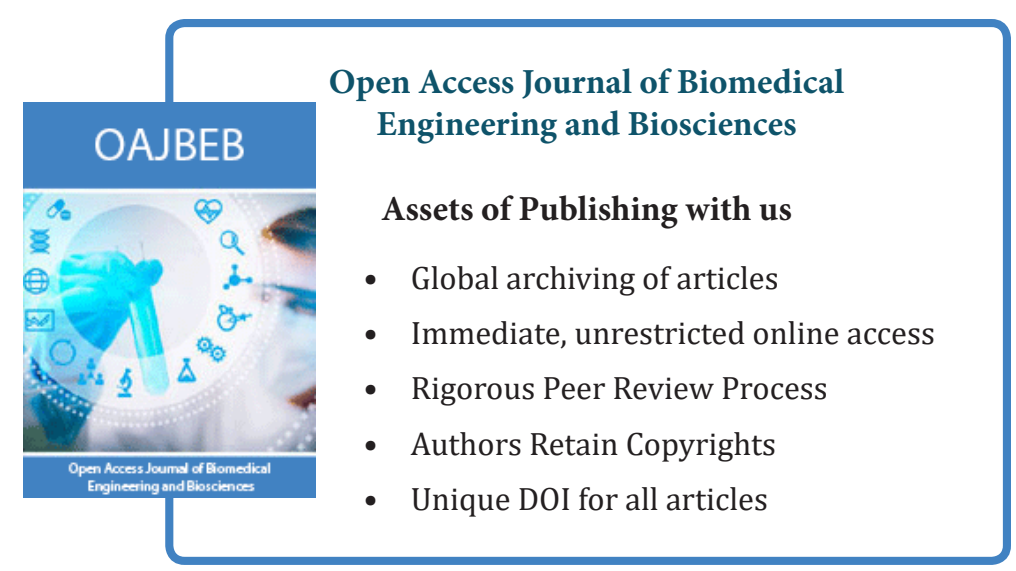

